\title{
Categories and dimensions in psychiatric diagnosis
}

\author{
JOHN WING
}

\section{THE TERMINOLOGY OF DIAGNOSIS}

The Oxford English Dictionary (paraphrased) defines 'diagnosis', in its medical context, as:

- the identification of a disease by careful investigation of symptoms and history, i.e. each category is derived from its symptoms, signs and course;

- the opinion (formally stated) resulting from such investigations, i.e. a choice from a list of named disease categories;

- the processes of research that lead to hard knowledge about dysfunctions, causes and pathology.

Many, if not most, disease concepts with a good provenance have actually developed in this way, beginning with clinical observations and insights that are later validated.

- The term 'disease' appears only rarely in the manuals of the two main systems of psychiatric diagnosis: ICD-10 and DSM-IV. The usage of ICD-10 is summarised in the following statement:

The term 'disorder' is used throughout the classification, so as to avoid even greater problems in the use of terms such as 'disease' and 'illness'. Disorder is not an exact term, but it is used here to imply the existence of a clinically recognizable set of symptoms or behaviours associated in most cases with distress and interference with personal functions».

Two points made in these manuals should be emphasised. One is that dimensions and categories are not mutually exclusive. Both are useful. The other is that 'diseases' or 'disorders' are not persons. Persons have many kinds of problem. Only some of these problems can be described in terms of one or more diagnoses or disorders, such as are the subject

Indirizzo per la corrispondenza: Professor J. Wing, College Research Unit, Royal College of Psychiatrists, 11 Grosvenor Crescent, London SWIX 7EE (UK).

Fax $(+44)$ 171-235.2954. of this review. As a result of making a diagnosis, some limited but useful suggestions can be made about causes, course, treatment or care for some of the clinical problems specified. In addition, those afflicted and their relatives can be given useful information (including what is not known) and introduced to others with the same or similar problems, some of whom who will be able to help where doctors cannot. Last, but by no means least, describing a category in specific terms, such as the algorithms of ICD-10 and DSM-IV, provides a standard of reference that can be improved upon or discarded. Diagnostic criteria must compete with each other, in particular by providing evidence that they are useful in theory or practice. Any new criteria must depart in clearly specifiable ways from the standard. Each element throughout this process can and should also be described and measured dimensionally.

These propositions seem so unexceptionable that it is difficult to understand why the issue of categories and dimensions raises such passions. Anyone interested in advancing knowledge must be able to move flexibly from one type of thinking to another. Clouds merge into each other but they can be classified. Ice and water are categories, temperature is a dimension; both concepts are useful but for different purposes. Arguments as to which perspective is 'right' are a waste of time. Nevertheless, passions are aroused; nowhere more so than in the case of 'schizophrenia'. Part of the passion is due to ideologies of various kinds (Laing, 1967; Szasz, 1961) but much of the problem is due to a simple semantic trap (Bentall et al., 1988; Scheff, 1966).

\section{DISEASE ENTITIES AND DISEASE THEORIES}

In everyday conversation the natural way to define a concept is from 'left to right'. For example, 
'Schizophrenia is...', on the left, followed on the right by a formula such as the algorithm in ICD-10 that defines it. This is often the way undergraduate and postgraduate medical students are taught, because it is a convenient method of memorising facts. Aristotle stated that the term to be defined «is the name of the essence of a thing", and this kind of definition is called 'essentialist'. It leads naturally to an assumption that the 'essence' is a 'disease entity'.

Karl Popper (1945) pointed out that scientific definitions, by contrast, are much less natural. The right-hand part of the formula (which might be the same algorithm for schizophrenia) is a technical specification designed to be useful in testing hypotheses. It carries no significance in itself and the term so defined is only a shorthand label for the specification. These are empirical, or 'right to left', definitions, intended to be tested and, in due course, replaced.

The two approaches can be discerned throughout the history of medicine. In various forms, notably the Galenic humoral theory, the essentialist type of definition was dominant for 2000 years (Cohen, 1961). Rote teaching of ICD-10 formulae (instead of using them as useful reference points that are likely to change as knowledge increases) could actually reactivate this historical trend, just as it is being superseded in psychiatry and much of the rest of medicine. The introduction of perverse incentives, such as the requirement to use such diagnoses in order to qualify for fees, would make matters worse.

Criticisms of 'schizophrenia' or of other diagnostic categories are therefore well placed if directed at concepts of 'disease entity' but not if they threaten the empirical value of using quantifiable concepts. Making a diagnosis should be equivalent to putting forward a testable hypothesis, whether in a consulting room, in a laboratory, or in a population. Moreover, every prescription for an individual patient is a kind of experiment.

\section{HIERARCHY IN PSYCHIATRIC DISORDERS}

The three ancient hierarchies divided mental faculties into conative (will), cognitive (thought) and affective (emotion). A fourth, motor abnormality, should be added. Each of these four areas of functioning can be impaired independently or together with any of the others. However, according to the international diagnostic systems, diagnoses at the time of first referral must be made according to a simple hierarchy of severity and response to treatment that conflates all four elements.

- At the top are cognitive disorders such as dementia which, at least in the early stages, can be associated with any other type of problem. For example, schizophrenic or severe depressive symptoms occurring in the course of Huntington's disease or temporal lobe epilepsy or severe mental retardation are discounted for the purpose of diagnosis.

- Next, conative, cognitive and motor disorders in the autistic spectrum, diagnosed on the developmental history, may co-exist with symptoms of disorders lower in the spectrum to the extent that the dominent abnormalities allow, without changing the diagnosis.

- First rank schizophrenic symptoms are next in precedence for diagnosis, above symptoms of affective disorders if both are present, as they commonly are.

- Bipolar affective symptoms in turn take precedence over unipolar depression, and both of these take precedence over autonomic phenomena.

- The position of obsessional disorders is uncertain but should probably be placed higher than the affective disorders and not classified at the level of panic and anxiety as is the tendency at present.

- Symptoms such as fatigue, worry and muscular tension are regarded as non-specific.

This hierarchy is generally non-reflexive, i.e. disorders higher up tend to be associated with symptoms characteristic of disorders lower down but not vice versa. This is particularly true of positive symptoms and is therefore most completely demonstrated in the acute phases of illness (Pakaslahti, 1986; Wing et al., 1974). The same hierarchy of approximate severity can be found in general population samples as well as in patients referred to specialists. Sturt (1981) demonstrated this independently of diagnosis. Motor abnormalities are now again becoming a focus for the attention of researchers. Frith (1992) for example, emphasises as did Kraepelin, the connection between catatonia and the will. Motor abnormalities can be present at any level but, as with the positive symptoms, there is a general gradient of severity from top to bottom. Thus psychiatric phenomena might usefully be regarded as continuously distributed along four basic dimensions, each representing a hierarchy of severity of abnormality in its own type of functioning. Current international diagnostic practice, however, is better represented as a series of cross-sections through the four dimensions, each 
slice manifesting a complex of symptoms, some positive and some negative. Both perspectives, longitudinal and cross-sectional, are legitimate and practical ways of investigating phenomena (Wing, 1978; 1995a). A combination of the two would be difficult but worth trying.

Systems such as SCAN/PSE-10 provide an opportunity for measurement 'bottom-up' (symptoms and dimensional scores), as well as for 'top-down' diagnoses for ICD-10, DSM-IV and other systems (Wing, 1995b; Wing et al., 1996). Because the clinical database is extensive, most other sets of diagnostic criteria could readily be supported. A recent thorough statistical study using PSE-9 data (Mulder-Hajonides, 1994) has suggested that a combination of classificatory and dimensional methods is most appropriate for taking these issues forward.

Perhaps ICD-11, in the next century, will be clinically multidimensional, thus supporting the representation of both cross-sectional and the longitudinal perspectives. It would be most unlikely, however, that the basic symptomatology that patients describe to doctors will differ substantially from what they experience now.

\section{REFERENCES}

Bentall R.P., Jackson, H.F. \& Pilgrim D. (1988). Abandoning the concept of schizophrenia. British Journal of Clinical Psycho$\log y$ 27, 303-324.

Cohen H. (1961). The evolution of the concept of disease. In Concepts of Medicine (ed. B. Lush). Pergamon: Oxford.

Frith C.D. (1992). The Cognitive Neuropsychology of Schizophrenia. Erlbaum: Hove.

Laing R.D. (1967). The Politics of Experience. Penguin: Harmondsworth.

Mulder-Hajonides W.R.E.H. (1994). Categories or Dimensions? The Use of Diagnastic Models in Psychiatry. Thesis University of Groningen, Faculty of Medicine.

Pakaslahti A. (1986). Principles and Practice of Diagnosing Schizophrenia. Social Insurance Institute: Helsinki.

Popper K.R. (1945). The Open Society and its Enemies. Routledge: London.

Scheff T.J. (1966). Being Mentally Ill. Aldine: Chicago.

Szasz T. (1961). The Myth of Mental Illness. Hoeber-Harper: New York.

Sturt E. (1981). Hierarchical patterns in the distribution of psychiatric symptoms. Psychological Medicine 11, 783-794.

Wing J.K. (1978). Reasoning about Madness. Chapter 3. Oxford University Press: Oxford.

Wing J.K. (1995a). Concepts of schizophrenia. In Schizophrenia. Chapter 1. (ed. S. R. Hirsch and D. Weinberger). Blackwell: Oxford (in press).

Wing J.K. (1995b). SCAN and the PSE tradition. Social Psychiatry and Psychiatric Epidemiology 30 (in press).

Wing J.K., Cooper, J.E. \& Sartorius N. (1974). The Measurement and Classification of Psychiatric Symptoms. Chapter 7. Cambridge University Press: Cambridge.

Wing J.K., Sartorius N. \& Üstün T.B. (1996). Diagnosis and Clinical Classification in Psychiatry. A Reference Manual for the SCAN System. Cambridge University Press: Cambridge (in press). 